\title{
Autoimmune Pancreatitis Presenting as Obstruction Jaundice
}

\author{
MA RAHMAN ${ }^{\mathrm{a}}$, IK DATTA ${ }^{\mathrm{b}}$, R BHUYIAN ${ }^{\mathrm{c}}$, TM BHUIYAN $^{\mathrm{d}}$, MM KABIR $^{\mathrm{e}}$, MN HAQUE$^{\mathrm{f}}$, \\ MG AZAMg, MA MAMOON ${ }^{\text {h }}$, MN MOHSIN ${ }^{\mathrm{i}}$
}

\begin{abstract}
Summary:
Autoimmune pancreatitis is a type of chronic pancreatitis characterized by an autoimmune inflammatory process in which prominent lymphocyte infiltration with associated fibrosis of the pancreas causes organ dysfunction.

Clinically it is very important to be aware of autoimmune pancreatitis because autoimmune chronic pancreatitis (AIP)
\end{abstract}

\section{Introduction:}

The term autoimmune pancreatitis was introduced by Yoshida et al. in 1995

a. Professor Md. Anisur Rahman FCPS (Medicine), Professor and Head of the Department of Medicine and Gastroenterology, BIRDEM Hospital and Ibrahim Medical College.

b. Dr. Indrajit Kumar Datta, FCPS (Medicine), MD (Gastroenterology) Registrar, Department of Gastroenterology. BIRDEM Hospital and Ibrahim Medical College

c. Dr. Rokanuzzaman Bhuyian, FCPS (Medicine), Student of thesis part, MD (Gastroenterology) BIRDEM Academy.

d. Dr. Tareq M. Bhuiyan FCPS (Medicine) Associate Professor Department of Gastroenterology., BIRDEM Hospital and Ibrahim Medical College.

e. Dr. Md. Mohsin Kabir, MD (Gastroenterology), Assistant Professor, Department of Gastroenterology., BIRDEM Hospital and Ibrahim Medical College

f. Dr. Md. Nazmul Haque, MD (Gastroenterology), Registrar, Department of Gastroenterology. BIRDEM Hospital and Ibrahim Medical College

g. Dr. Md. Golam Azam MD (Hepatology), Senior Research officer, BIRDEM Hospital

h. Dr. Md. Abdullah Al Mamoon MD (Gastroenterology), Senior Medical Officer, Department of Gastroenterology. BIRDEM Hospital.

i. Dr. Mostafa Noor Mohsin FCPS (Medicine), Student of thesis part, MD (Gastroenterology) BIRDEM Academy, Department of Gastroenterology, BIRDEM Hospital and Ibrahim Medical College. Address of correspondence

Address of Correspondence: Dr. Indrajit Kumar Datta, FCPS (Medicine), MD (Gastroenterology) Registrar, Department of Gastroenterology. BIRDEM Hospital and Ibrahim Medical College.

Received: 28 April 2010

Accepted: 20 September 2011 can clinically disguise as pancreaticobiliary malignancies, ordinary chronic or acute pancreatitis. ${ }^{1}$ Here we report a case of autoimmune pancreatitis presented with obstructive jaundice with initial diagnosis of carcinoma of head of the pancreas.

(J Bangladesh Coll Phys Surg 2011; 29: 231-234)

to describe the form of chronic pancreatitis that is associated with autoimmune manifestations revealed on laboratory, histologic and clinical testing. ${ }^{2}$ Autoimmune pancreatitis is rare.

Autoimmune pancreatitis is associated with hypergammaglobulinemia, histologic evidence of lymphoplasmacytic infiltration, coexistence of other autoimmune diseases and favorable response to steroid therapy, which are clinical evidence of autoimmunity. Autoantibodies against lactoferrin and carbonic anhydrase II have been identified as potential serologic markers of AIP. ${ }^{3,4}$ Patients with AIP present with a wide variety of symptoms- eg. obstructive jaundice and abdominal pain. Most symptoms associated with AIP are responsive to corticosteroid therapy. Occasionally patients present with symptoms related to extrapancreatic organ involvement. The Japan Pancreas society has proposed a diagnostic criteria that contains three items:(1) radiologic imaging, diffuse swelling of pancreas and segmental or diffuse irregular narrowing of the main pancreatic duct; (2) laboratory data, elevated IgG4 or detection of autoantibodies and (3) histopathologic examination, lymphoplasmacytic infiltration and fibrosis in the pancreas. ${ }^{5}$ For the diagnosis of AIP, all of the criteria should be present or criterion 1 together with either criterion 2 or 3 .

\section{Case Report:}

Mr. Hannan, a 56 years old diabetic, normotensive, non alcoholic, married businessman from Siddhirgonj, Dhaka presented with epigastric pain for 4 months which 
was deep, boring in nature with radiation to back, associated with nausea and vomiting. Pain usually worsened after meal and eased by leaning forward and taking analgesics. He also developed progressive jaundice with generalized itching for 2 months. His stool was pale. He had no history of fever, arthralgia, bleeding per rectum or vomiting of blood. He was diabetic for 6 months and on insulin. He is ex-smoker.

On examination he was mildly anaemic, moderately icteric. Scratch marks was present over different parts of body. Temperatre- $98^{\circ} \mathrm{F}$, Pulse: $80 / \mathrm{min}, \mathrm{BP}: 120 / 70$ mmHg, Respiratory rate: $14 / \mathrm{min}$. Abdomen examination revealed tenderness over epigastrium with no organomegaly and no other palpable lump. Other system examination revealed no abnormality. Liver function test was cholestatic in nature (serum bilirubin $6.7 \mathrm{mg} /$ dl, SGPT $235 \mathrm{U} / \mathrm{ml}$, SGOT $126 \mathrm{U} / \mathrm{ml}$, Serum Alkaline Phosphatase $823 \mathrm{U} / \mathrm{ml}$, serum total protien $72 \mathrm{gm} / \mathrm{L}$, Albumin $41 \mathrm{gm} / \mathrm{L}$.). Viral screen for hepatitis was negative (HBs Ag, Anti HEV IgM, Anti HCV). USG of whole abdomen showed that inhomogenous echotexture in head region of pancreas with mildly dilated common bile duct (CBD) and mildly distended Gall bladder; suggestive of $\mathrm{Ca}$ head of pancreas Pancreatitis. CT scan of upper abdomen showed large homogenous exophytic mass lesion measuring $3.9 \times 3.5 \mathrm{~cm}$ seen in the head of pancreas with mild prominence of pancreatic duct. Small sized (up to $2.1 \mathrm{~cm}$ ) lymph node seen in the periportal region suggesting regional lymphadenopathy. CT diagnosis was possible neoplasm of head of pancreas with infiltration to distal $\mathrm{CBD}$ and regional lymphadenopathy. With the working diagnosis of $\mathrm{Ca}$ head of pancreas ERCP was done. ERCP diagnosis was carcinoma of head of pancreas involving lower common bile duct. So endoprosthesis was implanted to decompress billiary tree. Patient responded well. His bilirubin came down to normal. Itching was less. Then for further evaluation CT guided FNAC of pancreatic head lesion was done and it revealed moderate cellular material containing pancreatic acinar cells, duodenal mucosal epithelium, a few polymorphs, lymphocytes and histiocytes in the background of blood. No malignant cells or granuloma seen.

He went to Tata Memorial Hospital, Mumbai, India where he was evaluated. Endosonography was done and head of the pancreas showed heterogenous echo texture. No definite hypo echoic lesion seen in the head region. Body
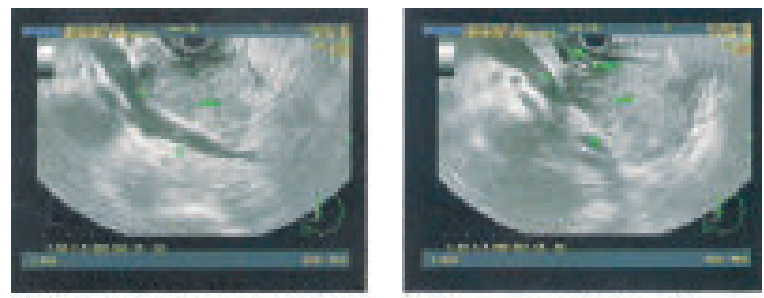

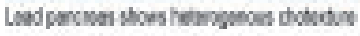

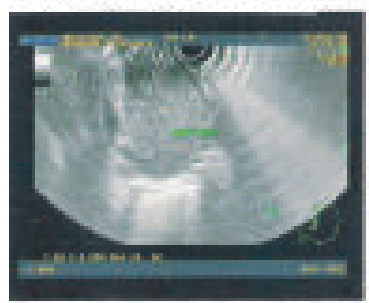

ClID seen with stent in stu
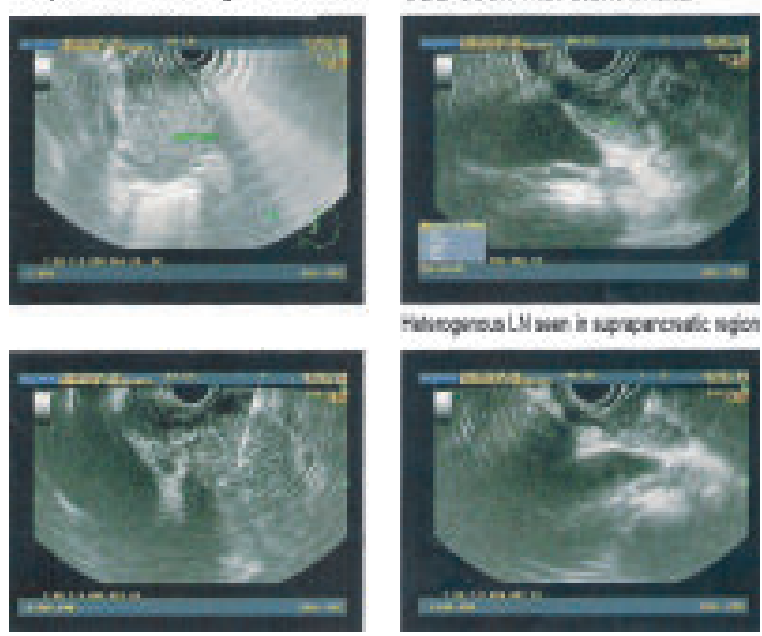

Fig: Endosonography showing heterogenous head of the pancreas, stent in $C B D$ and heterogenous lymph node in suprapancreatic region.

and tail of pancreas showed similar echogenicity. CBD showed uniformly thick wall. Several heterogenous lymph nodes were seen in the supra pancreatic region. The aetiology was likely to be inflammatory. EUS guided cytology: FNA from pancreatic mass showed that scattered honeycomb fragments and clusters of ductal cells exhibiting mild nuclear atypia were noted. The background showed scattered lymphocytes, bare nuclei and stromal fragments. No malignant cells observed. FNA from peripancreatic lymph nodes: the smears revealed stromal fragments, lymphohistiocytic clusters against a haemorrhagic background,also noted scattered mature and transformed lymphocytes along with benign granular cells.

Serum immunoglobulin G ( IgG): 970 mg/dl (Ref 600$1500 \mathrm{mg} / \mathrm{dl}$ )

Serum immunoglobulin G4 (IgG4): 5.69 gm/l (Ref 0.03$2.0 \mathrm{gm} / \mathrm{l})$

He was readmitted in our department with abdominal pain of previous type, loss of appetite, generalized weakness and weight loss. This time he was mildly 
anaemic otherwise normal general and systemic examination findings. SGPT $84 \mathrm{U} / \mathrm{ml}$, SGOT $83 \mathrm{U} / \mathrm{ml}$, ALK $314 \mathrm{U} / \mathrm{ml} \mathrm{S}$. Bilirubin $1.0 \mathrm{mg} / \mathrm{dl}$. S. amylase 25 u/l, S. Lipase $28 \mathrm{u} / 1$.

Total Cholesterol 298 gm/l, Triglyceride 198 gm/l. Fasting blood sugar $7.2 \mathrm{mmol} / \mathrm{l}, \mathrm{ABF} 11.6 \mathrm{mmol} / \mathrm{l}$. Along with premix insulin injection we started empiric treatment with tab. prednisolone $40 \mathrm{mg}$ daily after meal for initial 1 week followed by tapering of doses by $5 \mathrm{mg} /$ week. Follow up visit after 3 weeks patient was asymptomatic. $\mathrm{Hb} \% 12.9 \mathrm{gm} / \mathrm{dl}$, total $\mathrm{WBC}$ count 14500/cmm, Neutrophil 80\% Lymphocyte15\% ESR $20 \mathrm{~mm}$ in $1^{\text {st }}$ hour. SGPT- $70 \mathrm{U} / 1$, Alkaline Phosphatase$247 \mathrm{U} / 1$, and Blood sugar was controlled. He is now on regular follow up. He has no symptoms. He is getting Tab. Prednisolone $5 \mathrm{mg}$ daily as maintenance dose.

\section{Discussion:}

Autoimmune pancreatitis present with multiple symptoms, but severe abdominal pain or acute pancreatitis is unusual. In a large series, $63 \%$ of patients had jaundice and $35 \%$ of patients had abdominal pain. ${ }^{6}$ Patients rarely complained of typical severe abdominal pain of pancreatitis, and the major presenting symptom of many patients $(65 \%)$ was painless jaundice. Other symptoms of AIP included nonspecific mild abdominal pain (35\%) and weight loss (35\%). Diabetes mellitus is often noticed in AIP with reports ranging from $42 \%$ $76 \%$. ${ }^{7,8}$. Our patient presented with epigastric pain having characteristics of pancreatitis and progressive jaundice with generalized itching consistent with obstructive type.

The classic appearance of the pancreas on abdominal $\mathrm{CT}$ in patients with diffuse pancreatic involvement is sausage-shaped enlargement with homogeneous attenuation, moderate enhancement, and the peripheral rim of a hypo attenuation "halo". In long-standing autoimmune pancreatitis, involution of the pancreatic tail is almost always evident. Mild enlargement of the regional lymph nodes is also common. Focal pancreatic involvement is encountered more often in the head of the pancreas and typically appears as a low-attenuation or an isoattenuation mass. Therefore, the differential diagnosis of the focal form of autoimmune pancreatitis rather than pancreatic carcinoma can be very difficult on the basis of CT imaging only. In our case we initially diagnosed him as Carcinoma of head of páncreas and underwent ERCP and stenting. But CT guided FNAC excluded malignancy. EUS guided FNA from pancreatic mass and peripancreatic lymph nodes again excluded malignancy, rather it showed: Lymphohistiocytic clusters and scattered mature and transformed lymphocytes, the etiology was likely to be inflammatory.

According to the literature, IgG elevation or hypergmmaglobulinemia has been reported in $37-76 \%$ of the patients in AIP. IgG4, a subtype of IgG, levels have been reported to be able to distinguish AIP from other pancreatic disorders with a high sensitivity (95\%) and specificity (97\%). ${ }^{9}$ In our patient also IgG4 level was high.

In addition to the Japan Pancreas society another diagnostic criteria has been proposed by an Italian group: (1) histology and cytology, (2) the association with other postulated autoimmune diseases, and (3) the response to steroid therapy. ${ }^{5}$ Once a diagnosis of autoimmune pancreatitis has been made a treatment course with corticosteroids should be considered. Although the dramatic response to steroid is a well-known phenomenon in AIP, a detailed steroid schedule has not been fully established at the present time. The initial dose of corticosteroids (prednisone) should be $40 \mathrm{mg}$ daily for 1 week, followed by a taper of dose by $5 \mathrm{mg}$ per week. ${ }^{9,10}$ During the first 2 to 4 weeks of therapy, CT should be used to monitor the response. ${ }^{11,12}$ Laboratory abnormalities (such as elevated IgG4 levels, hypergammaglobulinemia, and presence of autoantibodies) may also show improvement with corticosteroid therapy. Extrahepatic biliary obstruction has also proved to be responsive to corticosteroid therapy. ${ }^{13} \mathrm{~A}$ poor response to corticosteroids should raise the question of pancreatic cancer or other forms of chronic pancreatitis. Although the vast majority of patients with autoimmune pancreatitis will readily respond to corticosteroids within a few weeks, a small subgroup may require maintenance therapy with prednisone at a dose of 5 to $10 \mathrm{mg}$ per day. ${ }^{14}$ The role of immunosuppressive agents has not been defined. On the basis of EUS guided FNA report which was in favour of inflammatory in nature and negative for malignancy and elevated IgG4 levels of serum; we diagnosed the patient as autoimmune pancreatitis.

\section{Conclusion:}

AIP is an immune-based systemic disease that should be diagnosed on the basis of imaging, histologic and 
serologic criteria. Its diagnosis is important, since AIP can mimic pancreatic cancer (as a pancreatic mass obstructing pancreatic-biliary duct) and respond so readily to corticosteroid therapy (CST). More use of pancreatic biopsy will aid in the diagnosis of AIP and provide a secure basis for the treatment with CST . The failure to differentiate AIP from malignancy may lead to unnecessary pancreatic resection for a benign disease which responds well to medical therapy

\section{References:}

1. Kyu-pyo Kim, M.D., Myung-Hwan Kim, M.D., Moon Hee, M.D., Sang SooLee, M.D., Dong Wan Seo, M.D., and Sung Koo Lee, Sang SooLee, M.D. Autoimmune Chronic Pancreatitis. Am J Gastrol 2004; 99:1605-16.

2. Yoshida K, Toki F, Takeuchi T, Watanabe S, Shiratori K, Hayashi N. Chronic pancreatitis caused by an autoimmune abnormality: proposal of the concept of autoimmune pancreatitis. Dig Dis Sci 1995; 40:1561-8.

3. Okazaki K, Uchida K, Ohana M, et al. Autoimmune-related pancreatitis is associated with autoantibodies and a Th1/Th2type cellular immune response. Gastroenterology 2000; 118:573-81.

4. Aparisi L, Farre A, Gomez-Cambronero L, et al. Antibodies to carbonic anhydrase and IgG4 levels in idiopathic chronic pancreatitis: relevance for diagnosis of autoimmune pancreatitis. Gut 2005; 54:703-9.

5. Peerson RK, Long Necker DS, Chery ST et al. Controversias in clinical pancreatology. Autoimmune pancreatitis: Does it exist? Pancreas 2007; 27:1-13
6. Kim KP, Kim MH, Song MH, Lee SS, Seo DW, Lee SK. Autoimmune chronic pancreatitis. Am J Gastroenterol 2004; 99:1605-16.

7. Horiuchi A, kawa S, Hamano H,et al. ERCP features in 27 patients with autoimmune pancreatitis. Gastroinest Endos 2002; 55:494-99.

8. Mackey IR, Gershwin ME. The nature of autoimmune disease. Semin Liver Dis1997; 17:3-11.

9. Hamano H, Kawa S, Horiuchi A et al. High serum IgG4 concentration in sclerosing cholangitis. N Engl J Med 2001;344:732-38.

10. Ito T, Nakano I, Koyanagi S, et al. Autoimmune pancreatitis as a new clinical entity: three cases of autoimmune pancreatitis with effective steroid therapy. Dig Dis Sci 1997; 42:1458-68.

11. Saito T, Tanaka S, Yoshida H, et al. A case of autoimmune pancreatitis responding to steroid therapy: evidence of histologic recovery. Pancreatology 2002; 2:550-6.

12. Kojima E, Kimura K, Noda Y, Kobayashi G, Itoh K, Fujita N. Autoimmune pancreatitis and multiple bile duct strictures treated effectively with steroid. J Gastroenterol 2003; 38:603-7.

13. Hirano K, Shiratori Y, Komatsu Y, et al. Involvement of the biliary system in autoimmune pancreatitis: a follow-up study. Clin Gastroenterol Hepatol 2003; 1:453-64.

14. Wakabayashi T, Kawaura Y, Satomura Y, Watanabe H, Motoo Y, Sawabu N. Longterm prognosis of duct-narrowing chronic pancreatitis: strategy for steroid treatment. Pancreas 2005; 30:31-9. 